\title{
Metacognição em grupos de Problem-based Learning (PBL)
}

\author{
Metacognition in Problem-based Learning (PBL) groups
}

\author{
Adriana Vargas* \\ Pontifícia Universidade Católica do Paraná \\ Evelise Maria Labatut Portilho** \\ Pontifícia Universidade Católica do Paraná
}

Resumo Aprendizagem em grupo é um processo que envolve a mobilização de funções cognitivas e desenvolve competências necessárias no decorrer da vida. A Problem-based Learning (PBL), uma proposta de aprendizagem em grupo, enfatiza o desenvolvimento cognitivo e pode potencializar a metacognição. Objetivou-se responder as questões: 1) a PBL é uma metodologia que envolve o desenvolvimento da metacognição? 2) pode-se relacionar os estudos da metacognição no contexto grupal à PBL? Realizou-se o estado do conhecimento do tema, entre 2010 a 2015, contendo os termos metacognition e Problem-based Learning, no portal de periódicos da CAPES. Oito pesquisas foram selecionadas por descreverem evidências empíricas da metacognição em grupos de PBL. A PBL pode ser aplicada aos estudos de aprendizagem em grupo e metacognição, por mobilizar conhecimentos, a tomada de consciência e o autocontrole.

PALAVRAS-CHAVE: Metacognição; Problem-based Learning; Aprendizagem em grupo.

Abstract Group learning is a process that involves cognitive functions mobilization and develops skills necessary throughout life. Problem-Based Learning (PBL), a proposal of group learning, emphasizes the cognitive development and may make the metacognition stronger. The objective was to answer the questions: 1) is $\mathrm{PBL}$ a methodology that involves metacognition development? 2) can we relate metacognition studies on group context to PBL? State of the art researches over the theme were realized, between 2010 - 2015, containing the terms metacognition and Problem-based Learning, in CAPES portal journals. Eight researches were selected because they describe empirical evidences of metacognition in PBL groups. PBL can be applied to studies group learning and metacognition, by mobilizing knowledge, awareness and self-control.

KEYWORDS: Metacognition; Problem-based Learning; Learning group. 


\section{Introdução}

As mudanças no contexto escolar contemplam o desenvolvimento de habilidades e competências dos sujeitos para serem bons aprendentes. Entretanto, ainda prevalece a negligência do aprender a aprender, a destruição inconsciente da resiliência do aprendiz e da reflexão (CLAXTON, 2005). Apesar do discurso do professor ser voltado para a mudança, constata-se que o modelo da prática docente hodierno é muito parecido com o tradicional, aquele que objetiva unicamente a transposição do conhecimento para o aluno. Nesse sentido, Cosme e Trindade (2010) observam que diante do modelo ou paradigma da instrução':

é de esperar que o acto de ensinar seja visto quer como um acto através do qual se difunde informação, quer como acto de imposição de normas e convenções exteriores aos sujeitos que aprendem, sem ter em conta tanto o seu estilo cognitivo, como os saberes de que estes são portadores (COSME; TRINDADE, 2010, p. 39).

Para superar este cenário, a alternativa apontada por Claxton (2005) levaria o professor, em suas estratégias de ensino, a não adotar soluções convencionais ou estilos triviais de estruturação de problemas, e sim, permitir aos alunos conhecer as próprias potencialidades ou fragilidades e seu repertório de estratégias de aprendizagem.

A metacognição possibilita desenhar o perfil de um aprendiz mais eficiente, que teria a habilidade de relacionar novas informações às já existentes, de selecionar as estratégias com maiores chances de sucesso, de planejar, monitorar e saber avaliar seus empreendimentos cognitivos (FLAVELL, 1979; NELSON; NARENS, 1994; JOU; SPERB, 2006; KIM; et. al, 2013). Características que condizem com o perfil profissional e competências (SCALLON, 2015) procuradas pelas organizações que, em decorrência das transformações da sociedade, mudaram também as relações de trabalho. A metacognição é, assim, uma importante ferramenta para a formação do indivíduo competente que, de acordo com Scallon (2015), é o indivíduo que sabe mobilizar um conjunto integrado de recursos internos em vistas de resolver uma família de situações problema. E a proposta da Problem-based Learning (PBL) ou aprendizagem baseada em problema se aproxima da demanda de formação nas escolas e universidades: por indivíduos competentes diante das mais variadas situações da vida.

A aprendizagem em grupos e a metacognição aparecem em pesquisas internacionais como em Kim et. al (2013) e, indiretamente, em estudos sobre programas de formação continuada de professores como em Araújo e Portilho (2014) e Portilho e Medina (2016) que apontam a necessidade de envolver a autorreflexão ao processo de aprendizagem do professor. Este artigo defende que a aprendizagem em grupo é um modelo propício para o desenvolvimento da metacognição, que parte da dimensão individual do processo de autorregulação para a dimensão grupal. Ainda, a atualidade do tema Problem-based Learning (PBL) em propostas metodológicas de ensino parece ser uma forma de proporcionar o desenvolvimento metacognitivo. Pretende-se, assim, responder a duas questóes: 1) a PBL é uma metodologia que envolve o desenvolvimento da metacognição? 2) pode-se relacionar os estudos da metacognição no contexto grupal à proposta da PBL? Para responder à essas questões recorreu-se ao estado do conhecimento do tema PBL e sua relação com a metacognição e aprendizagem em grupos. 


\section{Conhecimento metacognitivo e autorregulação em grupos}

A metacognição pode ser entendida como a possibilidade do sujeito tomar consciência e autorregular seus atos e pensamentos em relação ao próprio processo de conhecimento. Flavell (1979) foi um dos primeiros autores a considerar a metacognição como uma área específica de pesquisa. $\mathrm{O}$ autor propõe um modelo do monitoramento cognitivo, que ocorre por meio de ações e interações entre quatro classes de fenômenos: a) conhecimento metacognitivo; b) experiências metacognitivas; c) metas (ou tarefas); e d) ações (ou estratégias). Segundo ele, o conhecimento metacognitivo é o conhecimento do mundo, armazenado na memória de longo prazo, que compreende as pessoas como seres cognitivos com diferentes metas, ações e experiências metacognitivas. São conhecimentos ou crenças adquiridas por meio da experiência que o sujeito possui sobre seu próprio funcionamento, sobre os fatores ou variáveis que interagem para interferir no curso e no resultado dos empreendimentos cognitivos.

As experiências metacognitivas, segundo Flavell (1979), podem ser entendidas como sentimentos conscientes que surgem durante uma atividade cognitiva. São todas as experiências cognitivas ou afetivas conscientes que acompanham e estão relacionadas a qualquer função intelectual. As experiências metacognitivas estão relacionadas a tomada de consciência do momento que se está vivendo e do tipo de progresso que se fez num empreendimento cognitivo, podem auxiliar no controle de pensamentos e sentimentos sobre o próprio pensamento. As metas (ou tarefas) referem-se a objetivos de um empreendimento cognitivo. $\mathrm{E}$ as ações (ou estratégias) referem-se a comportamentos empregados para alcançar tais metas, como maneiras mais eficazes de se realizar uma atividade, algo importante para a autonomia do aprendiz.

Por meio da metacognição o aprendiz pode relacionar novas informações às já existentes, selecionar as estratégias com maior chance de sucesso, planejar, monitorar e saber avaliar seus empreendimentos cognitivos (JOU; SPERB, 2006). Em grupos de formação continuada com professores, segundo Portilho e Medina (2016), a abordagem metacognitiva incentiva aprender a aprender, pois requer a consciência e regulação das atividades cognitivas de seus participantes tendo como objetivo a transformação da prática pedagógica. De acordo com essas mesmas autoras, para o professor, a aprendizagem em grupo envolve a reflexão sobre suas habilidades e limitações no planejamento, monitoramento e avaliação de suas práticas de ensino com seus pares. $\mathrm{Na}$ pesquisa de Araújo e Portilho (2014), ao investigar a metacognição num grupo de professores de um programa de formação continuada, constataram que os itens relacionados à tomada de consciência de um questionário metacognitivo foram mais assinalados pelos integrantes do grupo, revelando a consciência de autorregulação provocada nas atividades grupais.

Kim et al. (2013) reconceituam a construção da metacognição considerando três níveis: o pensamento sobre o pensamento no nível individual, no nível social e ambiental. No nível individual, o aluno tem acesso a fontes internas para monitorar ou regular seus processos cognitivos. No entanto, isso ocasiona o que os autores denominaram de um paradoxo da metacognição; a metacognição é pessoal, mas não pode ser explicada exclusivamente por concepções individualistas. Uma maneira de resolver 
esse paradoxo é observar que, além de seus recursos psicológicos internos, os indivíduos também têm acesso a fontes externas que provocam o pensamento metacognitivo. Estas fontes incluem tanto as causas sociais, que vêm de outras pessoas que interagem com o indivíduo (nível social), e causas ambientais (nível ambiental), que vêm da interação com o ambiente em que se está aprendendo, como atividades de sala de aula ou a complexidade das tarefas. As fontes externas ao indivíduo informam falhas metacognitivas, como a ausência do comportamento de verificação da atividade. Por exemplo, uma pessoa ao falar em voz alta sobre o desenvolvimento de seu pensamento em torno de uma concepção particular de um problema pode fornecer feedback para o pensamento de outra pessoa sobre sua própria conceituação.

As fontes sociais que influenciam a metacognição são variáveis importantes na mudança de atitudes e crenças dos estudantes como solucionadores de problemas. Nesse sentido, metodologias de ensino e aprendizagem como a PBL, que envolvem interações com o ambiente de aprendizagem e atividades em grupo, favorecem o desenvolvimento da metacognição por tornar acessível fontes de conhecimento mais ricas potencialmente e proporcionar a superação das limitações individuais por meio de feedback e críticas de outros indivíduos. Para Giordan (1998), trabalhar em grupo induz uma variedade de ideias que muitas vezes são opostas, concepções vacilam e inicia-se uma dinâmica de modificação de ideias ou modelos de apreender o ambiente. Para ele, buscar em conjunto a proposta apropriada a um problema conduz a um distanciamento do mesmo, levando à reformulação de ideias, de raciocínios e à abstração. Pozo (2002) afirma que a aprendizagem em grupo favorece o surgimento de conflitos cognitivos entre os participantes por meio de comparação de pontos de vista, comparação dos planos elaborados pelos participantes, explicação (quando é preciso compartilhar com outros seus planos e estratégias). Os conflitos originados no grupo desvelam contradições implícitas no discurso dos participantes, colocando em dúvida o que é pensado a respeito de um tema, e obriga o sujeito a explicitar seu pensamento, argumentando e revendo suas crenças. O grupo também proporciona suporte ou apoio para resolver os problemas e alcançar os objetivos (individuais e coletivos), pois promove suporte para as dúvidas e respostas para as questões pertinentes.

Pode-se dizer que a aprendizagem em atividades grupais incentiva o desequilíbrio piagetiano, um mecanismo fundamental na promoção de reflexão e tomada de consciência. Piaget (1987) entende o processo de aquisição de novos conhecimentos como um processo de equilibração cognitiva que permite aos seres vivos assimilar as informações vindas do meio ambiente acomodando-as em estruturas mentais que são forjadas para refletir o mundo externo. Existe uma necessidade cognitiva de equilíbrio nos seres vivos, este equilíbrio se estabelece quando o indivíduo conhece seu meio ambiente de forma a prever os resultados de suas ações antes de executá-las. Mas, às vezes, as previsões não dão certo e o indivíduo se vê diante de novas situações com o meio ambiente, gerando um desequilíbrio. $\mathrm{O}$ processo de construção de conhecimento acontece quando o indivíduo age no sentido de alterar suas próprias estruturas cognitivas para acomodar os desequilíbrios e chegar, assim, a uma nova situação de 
equilíbrio momentânea, que será desfeita diante de um novo episódio, num ciclo de desenvolvimento, gerando novas e mais complexas estruturas cognitivas. O contexto grupal facilitaria essa ativação de conhecimentos e processos de aprendizagem para construir novos conceitos.

\section{PBL - Problem-based Learning: aprendizagem baseada em problema}

A Problem-based Learning (PBL) é uma proposta pedagógica iniciada no Canadá (McMaster University) nos anos 60 e, posteriormente, aplicada na Universidade de Maastrich (Holanda). PBL ou aprendizagem baseada em problema é um método de instrução específico que envolve a resolução colaborativa de problemas por meio de intensa discussão e pesquisa (SIEGEL, 2012). Foi desenvolvido pela primeira vez em programas de escola médica (BARROWS, 1988; HUNG; JONASSEN; LIU, 2008) e adaptado em outras áreas profissionais (como Direito, negócios), em universidades, escolas secundárias e primárias.

Conhecida como uma estratégia metodológica centrada no aluno, a PBL propõe a organização de temáticas em torno de problemas, objetivando a integração interdisciplinar ligando componentes teóricos e práticos, com ênfase no desenvolvimento cognitivo de seus participantes (RIBEIRO, 2008). Os problemas de estudo podem ser desafios acadêmicos (problemas relacionados a estruturação de temas de uma determinada área de estudo); cenários (simulação de contextos profissionais reais ou fictícios) ou problemas da vida real (envolvem soluções aplicáveis em seu contexto original). A PBL contempla o trabalho em grupo facilitado por tutores, e cabe ao professor incentivar a busca pelas informações.

Uma característica fundamental da PBL é seu caráter formativo - à medida que estimula uma atitude ativa do aluno em busca do conhecimento - e não meramente informativo, como é o caso da prática pedagógica tradicional (RIBEIRO, 2008). Ao contrário do ensino tradicional no qual os alunos aprendem conteúdos e, em seguida, resolvem problemas, a PBL começa com os problemas (SIEGEL, 2012). Esta abordagem favorece a compreensão do porquê estudar um determinado conteúdo e como a teoria é aplicada na prática (BARROWS, 1996; GALLAGHER et al., 1995).

Segundo Collins, Brown e Newman (1987), a PBL tem uma configuração semelhante à aprendizagem cognitiva. No processo de aprendizagem os sujeitos se envolvem em tarefas reais, enquanto o professor ou coordenador torna os processos de pensamento tácitos em explicações explícitas. Da mesma forma, em PBL, os sujeitos resolvem problemas em grupos e são guiados por um facilitador que modela a resolução do problema, perguntando sobre os processos e desafiando os participantes a pensar e usar diversos recursos para resolver a situação problema.

A PBL também é um meio para proporcionar a reflexão profissional. Estudos demonstraram que professores aprenderam a tomar decisões relativas ao contexto profissional, ao invés de serem receptores passivos de informação, após a participação em grupos de PBL (VALLI, 1997). A PBL também auxilia os sujeitos a controlar 
a sua compreensão e reconhecer quando têm uma lacuna no próprio conhecimento (CHI; BASSOCK, 1989). Como é uma atividade desenvolvida em pequenos grupos, a resolução de problemas depende de compartilhamento de explicações e feedback, ou seja, responder de forma construtiva durante as interações do grupo, promovendo a retroalimentação (WEBB; MASTERGEORGE, 2003).

Estudos como os de Dods (1997) e Newble e Clarke (1986) mostraram que a PBL é eficaz na promoção de certos tipos de aprendizagem. Como foi observado pelos autores, os participantes das pesquisas ganharam maior aprofundamento na compreensão do conteúdo do que os métodos tradicionais de ensino, que se concentram na amplitude do conhecimento. O estudo de Mayo et al. (1993) apontou para o desenvolvimento de habilidades metacognitivas quando tutores de PBL de alunos de medicina ajudaram a promover as habilidades de raciocínio clínico necessárias a um médico especialista.

A PBL fundamenta-se na motivação epistêmica por meio de colocações e discussões sobre a busca da resolução do problema, sendo uma aprendizagem em grupo pautada na metacognição e em fatores sociais, alguns estudos apontam, especificamente, para a relação entre esta estratégia pedagógica e o desenvolvimento de habilidades metacognitivas, como será visto adiante.

\section{Metodologia}

Este artigo foi construído a partir do mapeamento de pesquisas sobre o estado do conhecimento em metacognição em grupos de PBL. Foi realizado um levantamento bibliográfico em revistas científicas no portal de periódicos da CAPES utilizando como critérios de busca os termos metacognition e Problem-based Learning, no período de 2010 a 2015, com objetivo de localizar pesquisas recentes sobre o tema. Neste mapeamento foram encontrados 92 estudos. Realizou-se a leitura dos resumos dos 92 artigos tendo como objetivo identificar as pesquisas que relatavam evidências do desenvolvimento da metacognição em grupos de PBL. A partir da leitura dos artigos, identificou-se referências de pesquisas das décadas de 90 e 2000 que também traziam evidências da relação entre metacognição e aprendizagem em grupos de PBL. Após esta análise, oito pesquisas foram selecionadas porque relatavam evidências empíricas da relação entre atividades em grupos de PBL e o desenvolvimento metacognitivo.

\section{Resultados e discussão}

As contribuições das pesquisas sobre a relação entre a proposta de PBL e o desenvolvimento da metacognição foram resumidas no Quadro 1. As pesquisas foram desenvolvidas em países como USA, Alemanha, China, Canadá e Japão. O estudo menos recente, de Hmelo, Gotterer e Bransford (1997), foi publicado da década de 90, quando os autores levantaram a hipótese que o desenvolvimento de características da metacognição estariam envolvidas na resolução de problemas. A pesquisa foi desenvolvida com grupos de estudantes de medicina que utilizavam protocolos de resolução de problemas para registrar as estratégias e hipóteses de resolução dos casos clínicos. A 
partir de 2004, os estudos relatam observações realizadas com grupos de PBL objetivando avaliar características da metacognição.

Quadro 1 - Resumo das contribuições e resultados das pesquisas sobre PBL e o desenvolvimento da metacognição

\begin{tabular}{|c|c|c|}
\hline Autor(es) & Ano & \begin{tabular}{|c|} 
Resultados / contribuições \\
\end{tabular} \\
\hline $\begin{array}{l}\text { Hmelo, Gotterer } \\
\text { e Bransford }\end{array}$ & 1997 & $\begin{array}{l}\text { Os processos envolvidos na resolução de } \\
\text { problemas devem levar ao desenvolvimento de } \\
\text { características da metacognição }\end{array}$ \\
\hline Hmelo-Silver & 2004 & $\begin{array}{l}\text { A PBL pode revelar-se particularmente dificil } \\
\text { para aqueles que tendem a ter dificuldade em } \\
\text { aplicar estratégias metacognitivas }\end{array}$ \\
\hline Lan et. al & 2010 & $\begin{array}{l}\text { A PBL auxilia os alunos a desenvolver um } \\
\text { conhecimento flexível e habilidades eficazes de } \\
\text { resolução de problemas, além de aumentar a } \\
\text { transferência de conceitos e conhecimentos } \\
\text { para novos problemas }\end{array}$ \\
\hline $\begin{array}{l}\text { Downing, Ning e } \\
\text { Shin }\end{array}$ & 2010 & $\begin{array}{l}\text { Melhorias em metacognição para o grupo de } \\
\text { estudantes que participaram da PBL } \\
\text { PBL exige do estudante monitoramento e } \\
\text { orientação do processo de resolução de } \\
\text { problemas }\end{array}$ \\
\hline Siegel & 2012 & $\begin{array}{l}\text { Os prováveis componentes da metacognição } \\
\text { em grupos seriam: consciência metasocial } \\
\text { sobre a expertise de outro membro do grupo; } \\
\text { monitoramento da compreensão; e } \\
\text { monitoramento de processo }\end{array}$ \\
\hline Stefanou et al. & 2013 & \multirow{2}{*}{$\begin{array}{l}\text { Identificaram niveis mais elevados de } \\
\text { metacognição em alunos em atividades de PBL }\end{array}$} \\
\hline Schauber et al. & 2015 & \\
\hline Lajoie et al. & 2015 & $\begin{array}{l}\text { Aumento das estratégias metacognitivas de } \\
\text { monitoramento, avaliação e elaboração em } \\
\text { sessões de PBL } \\
\text { Estágios de autorreflexão nas sessões de PBL } \\
\text { Forte ligação entre ações de co-regulação de } \\
\text { um grupo e estratégias metacognitivas de } \\
\text { planejamento }\end{array}$ \\
\hline
\end{tabular}

De acordo com Hmelo, Gotterer e Bransford (1997), por sua própria natureza, a PBL requer uma maneira diferente de usar o conhecimento para resolver problemas, e é esse "funcionamento" que envolve os processos metacognitivos. Consequentemente, como a PBL usa casos reais ou problemas como ponto de partida, os processos envolvidos na resolução destes problemas devem levar ao desenvolvimento de características da metacognição - como os conhecimentos são aplicados, o conhecimento sobre o quanto pode ser aprendido, que tipos de estratégias usar - um conjunto de procedimentos heurísticos gerais que envolvem planejamento, definição de metas e feedback dos processos (FRESE; STEWART; HANNOVER, 1987). 
$\mathrm{Na}$ busca por evidências empíricas, Hmelo-Silver (2004) reviu pesquisas que apontavam a PBL como fator que auxilia o desenvolvimento de conhecimento flexível; habilidades eficazes de resolução de problemas; habilidades de aprendizagem autodirigidas; capacidades de colaboração eficazes; e motivação. A autora concluiu que a atividade proposta pela PBL pode revelar-se particularmente difícil para aqueles que tendem a ter dificuldade em aplicar estratégias metacognitivas, entendendo, assim, o movimento das estratégias metacognitivas para resolução dos problemas em grupos de trabalho.

Lan et al. (2010) observaram que a PBL pode reduzir,inicialmente, os níveis de aprendizagem, mas por um período maior de atividade pode promover aumento da retenção de conhecimento. Os pesquisadores realizaram um estudo com 28 estudantes de uma escola primária em Taipei na China e aplicaram diferentes instrumentos, como discussões em grupo; atividades de resolução de problemas; um teste chamado Assessment of the Computational Estimation Abilities of Elementary School Students (CEA Assessment) que avaliou estratégias metacognitivas; uma plataforma eletrônica chamada Group Scribbles (GS), que permitiu geração, coleta e agregação de ideias por meio de um espaço compartilhado de anotações gráficos e textos dos participantes da pesquisa; observações e gravações em vídeo e estimativa computacional de dados. Para eles, as atividades em grupo de PBL auxiliam no desenvolvimento metacognitivo dos alunos, a desenvolver um conhecimento flexível e habilidades eficazes de resolução de problemas, além de aumentar a transferência de conceitos e conhecimentos para novos problemas. A proposta também elevaria o interesse do aluno pelo conteúdo das aulas e desenvolveria habilidades de colaboração no grupo (NORMAN; SCHMIDT, 1992; HMELO-SILVER, 2004).

Downing, Ning e Shin (2011) examinaram a eficácia da PBL no ensino superior com base em uma amostra de estudantes do primeiro ano de dois programas de uma Universidade de Hong Kong ( $\mathrm{n}=132)$. Foi aplicado um inventário, Learning and Study Strategies Inventory (LASSI), para mensurar a metacognição, entre o início e o fim dos primeiros 15 meses em cada programa. Após 15 meses e três semestres de estudo em diferentes ambientes os pesquisadores observaram melhorias no desenvolvimento metacognitivo para o grupo de estudantes que participaram da PBL. Segundo os pesquisadores, a PBL pode ser desenvolvida em diversas formas, no entanto, todas exigem do estudante monitoramento e orientação do processo de resolução de problemas, e conceitos e processos resgatados da memória para dar suporte ao problema atual ${ }^{2}$. Também propõem que a PBL deve ser idealmente adaptada para o desenvolvimento mais efetivo da metacognição na graduação do que as técnicas alternativas não baseadas em problemas. Para eles, atuar num ambiente de aprendizagem não tradicional, proporcionado pela $\mathrm{PBL}$, aumenta o uso da metacognição porque o aluno não pode invocar o conhecimento "rotineiro" ou "automático" para a resolução de problemas.

Como a proposta da PBL envolve a aprendizagem em grupo, parece oportuno trazer o estudo de Siegel (2012) para esta discussão. A pesquisa foi realizada com 67 alunos do quarto ano de graduação de um programa de certificação de professores matriculados no curso de Psicologia da Educação e 26 voluntários. Durante o pro- 
grama foram realizadas gravações em vídeos das sessões de PBL, escrita de textos e estudo de casos em vídeo de situações reais de instrução e resolução de problemas, e disponibilizado uma web site com cases, material adicional para consulta, problemas e projetos, discussões e network, vídeos de cases, links e fontes de pesquisas para os participantes. A autora criou uma estrutura para a conceitualização da metacognição em grupos, descrevendo prováveis componentes da metacognição em ambiente grupal, sendo eles: consciência metasocial sobre a expertise de outro membro do grupo, monitoramento da compreensão e monitoramento de processo. A consciência metasocial possibilita identificar quem ou qual integrante do grupo obtém o conhecimento necessário. É a consciência de si, dos outros, do grupo, e dos recursos do grupo - que são públicos. O monitoramento da compreensão refere-se ao autoconhecimento e ao conhecimento referente ao outro, o conhecimento público. Um subtipo encontrado para o monitoramento da compreensão é a identificação da necessidade de conhecimento, ou seja, quando as lacunas no conhecimento de um grupo tornam-se visíveis para todo o grupo. E o processo de monitoramento está ligado ao monitoramento do grupo publicamente, tendo um subtipo, mudanças de objetivos, que possibilitam ajustar as metas do grupo.

Sendo a PBL uma atividade realizada em grupo, acredita-se que os componentes da metacognição, descritos por Siegel (2012), estejam presentes no desenvolvimento de grupos de PBL. Na resolução dos problemas, as estratégias metacognitivas são socializadas quando explicitadas pelos integrantes, podendo ser ampliadas e aperfeiçoadas com a contribuição de cada pessoa na interação grupal.

As pesquisas de Stefanou et al. (2013) e Schauber et al. (2015) identificaram níveis mais elevados de elaboração, pensamento crítico e metacognição em alunos em atividades de PBL. A equipe de Stefanou et al. (2013) aplicou três questionários, o Motivated Strategies for Learning Questionnaire (MSLQ), o Learning Climate Questionnaire (LCQ) e o Perceived Autonomy-Supportive Climate Questionnaires developed num grupo de 77 estudantes de graduação de duas universidades privadas do nordeste dos Estados Unidos. Schauber et al. (2015) utilizaram uma amostra maior, 1.646 estudantes de uma tradicional Universidade de medicina de Berlim, os autores desenvolveram e aplicaram um questionário composto por dados demográficos básicos e auto relatos dos estudantes. As questões do questionário incluíram percepção do ambiente de aprendizagem, crenças de auto eficácia, efeito positivo de estudo, apoio social, indicadores de aprendizagem autorregulada, e desempenho acadêmico (avaliado por meio de testes de progresso).

Lajoie et al. (2015), com objetivo de examinar a relação entre a metacognição e as atividades de um grupo de PBL, descreveram os resultados de duas sessões projetadas para ajudar a quatro estudantes de medicina (2 do Canadá, 2 de Hong Kong) a entender os princípios da condução de uma entrevista médica para dar más notícias ao paciente. $\mathrm{O}$ ambiente de instrução consistiu de cinco módulos online por meio do software de conferência web Adobe Connect 9. Foi adotada uma abordagem com métodos exploratórios mistos para análise dos dados, com mineração de padrões sequenciais, estudo de caso e gravação de vídeos. Um estudante (como facilitador) liderava as discussões e outro era responsável pelas anotações e registros da atividade, 
enquanto os dois alunos restantes continuavam a discussão em grupo. No início do primeiro dia de PBL, as estratégias mais frequentes foram orientação o e planejamento. Já no final do primeiro dia, as estratégias metacognitivas eram mais direcionadas para avaliação e elaboração. $\mathrm{Na}$ segunda sessão, foram observados mais movimentos em estratégias de regulação, os alunos começaram a discussão de uma forma mais ativa e organizada e participaram na introdução de ideias, sendo capazes de focar em demandas das tarefas atribuídas. Com o avanço da discussão do grupo, os pesquisadores notaram maior monitoramento, avaliação e elaboração pelos integrantes. Planejamento foi a estratégia metacognitiva que ocorreu com mais frequência em ambas as sessões, uma vez que o problema girava em torno de situações futuras, dar retorno a um paciente sobre más notícias.

Na segunda metade das sessões de PBL do estudo de Lajoie et al. (2015) os alunos tendiam a ser mais reflexivos e também foram observados estágios de autorreflexão. $\mathrm{O}$ estudo concluiu que existe uma forte ligação entre as ações de co-regulação de um grupo e estratégias metacognitivas de planejamento. $\mathrm{O}$ termo co-regulação foi baseado na teoria sócio construtivista de Vigotski (1998), em que as funções psicológicas superiores são internalizadas por meio da interação social. A co-regulação abrange a colaboração de membros do grupo como um processo de transição para a autorregulação, sendo o ambiente social um apoio para a aprendizagem individual. Os membros do grupo podem atuar na regulação social da aprendizagem uns dos outros, além da própria autorregulação. Como visto, os alunos que participaram das sessões de PBL da pesquisa de Lajoie et al. (2015) tendiam a realizar um ciclo ${ }^{3}$ completo de estratégias metacognitivas que levavam a atividades de compartilhamento metacognitivo e co-regulação do grupo.

\section{Considerações finais}

Entende-se que a organização tradicional do currículo escolar por disciplinas gera a fragmentação do conhecimento. A palavra disciplina, com origem no latim, refere-se a ordem, organização. A visão globalizante do currículo, como adotada pela proposta da PBL, prevê a articulação do conhecimento de maneira interdisciplinar e multidisciplinar, levando a estratégias de ensino que atendam aos diferentes estilos de aprendizagem, contextos e perfis dos alunos. As propostas de aprendizagem em grupos de PBL contempla a perspectiva da complexidade e tem como foco a aprendizagem do sujeito, contemplando as dimensões cognitiva, metacognitiva e procedimental da aprendizagem.

A aprendizagem em grupos parece ser uma proposta eficaz no desenvolvimento da metacognição, como defendido por Kim et. al (2013). As estratégias metacognitivas acionadas pelos membros de um grupo operam como um todo para acompanhar conjuntamente e regular o processo cognitivo para o objetivo em comum. Para tanto, a comunicação é um fator crucial, a socialização de estratégias metacognitivas estão condicionadas a explicitação dos processos internos pelos membros do grupo que, de forma conjunta, realiza um ciclo metacognitivo de planejamento, execução, acompanhamento e avaliação. É inevitável a aproximação das pesquisas sobre a aprendizagem em grupos e o desenvolvimento da metacognição à proposta da PBL. 
No entanto, a efetivação dessa proposta está condicionada à superação de desafios no cenário da educação brasileira. Adotar a proposta da PBL requer um novo perfil docente, com conhecimento prévio sobre o tema e abertura para novas estratégias de ensino. O que se percebe nas escolas e universidades é a resistência do professor ou a insistência em práticas tradicionais de ensino. A esse novo papel do professor implica um redimensionamento dos cursos de licenciaturas e da formação continuada desses profissionais, de forma a contemplar uma perspectiva da aprendizagem problematizadora e reflexões sobre uma prática comprometida com as mudanças de concepções de ensino. Outro desafio a ser superado refere-se às poucas disciplinas com fundamentação da neurociência e psicologia cognitiva nos currículos de formação de professores, entendidos como conhecimentos prévios para o planejamento e acompanhamento das ações de ensino e aprendizagem.

Entende-se como outro desafio desta proposta o direcionamento de toda organização curricular de uma instituição que venha a adotar a PBL como estratégia de ensino. A PBL volta-se para a integração curricular das disciplinas sendo necessário mobilizar não apenas o corpo docente, mas toda a instituição de ensino para um trabalho integrado. Diante disso, os caminhos a serem percorridos para efetivar uma proposta de PBL na educação passam por mudanças estruturais na formação do professor e levam às reflexões do corpo docente sobre o papel do professor e seu compromisso com o ensino problematizador.

A PBL é uma metodologia propícia para o desenvolvimento da metacognição, visto que exige a mobilização de conhecimentos e estratégias metacognitivas pelo grupo na resolução de problemas. Sugere-se mais pesquisas no contexto das escolas brasileiras articulando estes dois temas, metacognição e grupos de PBL.

\section{Referências}

ARAÚJO, D. F.; PORTILHO, E. M. L. As contribuições da metacognição na formação continuada de professores: uma experiência rumo à aprendizagem e ao ensino. 2014. 192 f. Dissertação (Mestrado) - Pontifícia Universidade Católica do Paraná, Curitiba, 2014 Disponível em: $\langle$ http://www.biblioteca.pucpr.br/tede/tde_busca/arquivo.php?codArquivo=2857>. Acesso em: 29 set. 2016.

BARROWS, H. S. The tutorial process. Southern Illinois University School of Medicine, Springfield, IL. 1988. In: VELLA, F. The tutorial process: By HS Barrows, pp 63. Southern Illinois University School of Medicine, Springfield, IL. 1989. ISBN 0-931369-22-3. Biochemical Education, 17: 161-162. Disponível em: <http://onlinelibrary.wiley.com/doi/10.1016/03074412(89)90116-7/pdf> Acesso em: 5 jul. 2016.

H. S. Problem-based learning in medicine and beyond: a brief overview. In: WILKERSON, L; GIJSELAERS, W. H. (Eds.) Bring problem-based learning to higher education: theory and practice. New Directions for Teaching and Learning, vol 68, 1996, pp 3-12. Disponível em: <http://onlinelibrary.wiley.com/doi/10.1002/tl.37219966804/abstract> Acesso em: 5 jul. 2016.

CHI, M.T. H.; BASSOCK, M. Learning from examples via self-explanations. In: RESNICK, L. B. (ed) Knowing, learning, and instruction: essays in honor of Robert Glaser. Erlbaum, Hillsdale, pp 251-282 1989. Disponível em: <http://chilab.asu.edu/papers/ChiBassok8.pdf> Acesso em: 20 jul. 2016. 
CLAXTON, G. O desafio de aprender ao longo da vida. - Porto Alegre: Artmed, 2005.

COLLINS, A.; BROWN, J. S.; NEWMAN, S. E. Cognitive apprenticeship: Teaching the crafts of reading, writing, and mathematics. Children's Research Center, Technical Report No. 403, January p1-27 1987. Hillsdale, NJ: Lawrence Erlbaum Associates. Disponível em: $<$ https://www.ideals.illinois.edu/bitstream/handle/2142/17958/ctrstreadtechrepv01987i00403_ opt.pdf?sequence=1> Acesso em: 20 jul. 2016.

COSME, A.; TRINDADE, R. AVALIAR AS ESCOLAS: PARA QUÊ E PORQUÊ? In: SILVA, J. N. P.; TEIXEIRA, F.; NASCIMENTO, J. ELO 17 Auto- avaliação das Escolas e Processos de Auto-monitorização. Revista ELO 17 - setembro de 2010 (33-40). Disponível em: http://cffh.pt/userfiles/files/ELO\%2017.pdf\#page=33 Acesso em: 29 set. 2016.

DODS, R. F. An action research study of the effectiveness of problem-based learning in promoting the acquisition and retention of knowledge. Journal for the Education of the Gifted, v20 n4 p423-37 Sum 1997. Disponível em: <http://eric.ed.gov/?id=EJ553977> Acesso em: 20 jul. 2016.

DOWNING, K.; NING, F.; SHIN, K. Impact of problem-based learning on student experience and metacognitive development. Multicultural Education \& Technology Journal, v5 n1 p55-69 2011. Disponível em: <http://eric.ed.gov/?id=EJ923761> Acesso em: 8 jul. 2016.

FLAVELL, J. H. Metacognition and Cognitive Monitoring. American Psychologist, Vol 34(10), Oct 1979, 906-911. Disponível em: <http://psycnet.apa.org/index.cfm?fa=buy.optionToBuy\&id=1980-09388-001> Acesso em: 14 jul. 2016.

FRESE, M.; STEWART, J.; HANNOVER, B. Goal orientation and planfulness: Action styles as personality concepts. Journal of Personality and Social Psychology, Vol 52(6), Jun 1987, 1182-1194. Disponível em: <http://psycnet.apa.org/psycinfo/1987-28181-001> Acesso em: 8 jul. 2016.

GALLAGHER, S. A. et. al. Implementing problem-based learning in Science Classrooms. School Science and Mathematics 95(3):136-146, 1995. Disponível em: <http://onlinelibrary. wiley.com/doi/10.1111/j.1949-8594.1995.tb15748.x/abstract> Acesso em: 5 jul. 2016.

GIORDAN, A. Aprender. Instituto Piaget. - Av. João Paulo II, Lote 544, 2. - Lisboa. Éditions Belin, 1998.

HMELO, C. E.; GOTTERER, G. S.; BRANSFORD, J. D. A theory-driven approach to assessing the cognitive effects of PBL. Instructional Science November 1997, Volume 25, Issue 6, pp 387-408. Disponível em: <http://link.springer.com/article/10.1023/A:1003013126262〉 Acesso em: 8 jul. 2016.

HMELO-SILVER, C. E. Problem-based learning: What and how do students learn? Educational Psychology Review September 2004, Volume 16, Issue 3, pp 235-266. Disponível em: $<$ http://link.springer.com/article/10.1023/B:EDPR.0000034022.16470.f3> Acesso em: 8 jul. 2016.

HUNG, W; JONASSEN, D. H.; LIU, R. Problem based learning. In: SPECTOR, J. M. et. al (Eds.) Handbook of research on educational communications and technology. Taylor \& Francis, New York, 2008 pp 486-498. Disponível em: <http://aect.org/edtech/edition3/er5849x_c038. fm.pdf> Acesso em: 5 jul. 2016.

JOU, G. I.; SPERB, T. M. A metacognição como estratégia reguladora da aprendizagem. Psicol. Reflex. Crit., Porto Alegre, v. 19, n.2, p. 177-185, 2006. Disponível em: <http://www.scielo. br/scielo.php?script=sci_arttext\&pid=S0102-79722006000200003\&lng=en\&nrm=iso >. Acesso em: 17 nov. 2016.

KIM, Y. R. et. al. Multiple levels of metacognition and their elicitation through complex problem-solving tasks. The Journal of Mathematical Behavior Volume 32, Issue 3, September 2013, Pages 377-396. Disponível em: <http://www.sciencedirect.com/science/article/pii/ 
S0732312313000412> Acesso em: 11 jul. 2016.

LAJOIE, S. P. et. al. The role of regulation in medical student learning in small groups: Regulating oneself and others' learning and emotions. Computers in Human Behavior. Volume 52, November 2015, Pages 601-616. Disponível em: <http://www.sciencedirect.com/science/ article/pii/S0747563214006918> Acesso em: 6 jul. 2016.

LAN, Y. et. al. Mobile-Device-Supported Problem-Based Computational Estimation Instruction for Elementary School Students. Educational Technology \& Society, v13 n3 p55-69 2010. Disponível em: <http://eric.ed.gov/?id=EJ899862> Acesso em: 8 jul. 2016.

NELSON, T. O.; NARENS, L. Why Investigate Metacognition? In J. Metcalfe and A. P. Shimamura (Eds.) Metacognition. Cambridge, Mass.: The MIT Press, 1994. Pp.1-26. Disponível em: http://www.imbs.uci.edu/ lnarens/narens.html. Acesso em: 24 de nov de 2015.

MAYO, P. et. al. Student perceptions of tutor effectiveness in a problem-based surgery clerkship. Teaching and Learning in Medicine: An International Journal Volume 5, Issue 4, 1993. Disponível em: <http://www.tandfonline.com/doi/abs/10.1080/10401339309539628> Acesso em: 21 de jul de 2016.

NEWBLE, D.I., CLARKE, R.M. The approaches to learning of students in a traditional and in an innovative problem-based medical school. Medical Education Volume 20, Issue 4, pages 267273, July 1986. Disponível em: <http://onlinelibrary.wiley.com/doi/10.1111/j.1365-2923.1986. tb01365.x/abstract> Acesso em: 20 de jul de 2016.

NORMAN, G.R., SCHMIDT, H.G. The psychological basis of problem-based learning: a review of the evidence. Acad Med. 1992 Sep;67(9):557-65. Disponível em: <http://www.ncbi. nlm.nih.gov/pubmed/1520409> Acesso em: 8 jul. 2016.

PIAGET, J. O nascimento da inteligência na criança. 4. ed. Rio de Janeiro: Guanabara, 1987.

PORTILHO, E. M. L.; MEDINA, G. B. K. Metacognition as Methodology for Continuing Education of Teachers. Creative Education, 7, 1-12, 2016. Disponível em: < http://www.metacognicao.com.br/wp-content/uploads/2016/01/Clique-Aqui-2.pdf> Acesso em: 29 set. 2016.

POZO, J. I. Aprendizes e mestres: a nova cultura da aprendizagem / Juan Ignacio Pozo; trad. Ernani Rosa. - Porto Alegre: Artmed Editora, 2002.

RIBEIRO, L. R. C. Aprendizagem baseada em problemas (PBL): uma experiência no ensino superior (online). São Carlos: EduFSCar, 2008. ISBN 978-85-7600-297-o. Disponível em: <http://books.scielo.org/id/w57z2> Acesso em: 5 jul. 2016.

SCALLON, G. Avaliação da aprendizagem numa abordagem por competência. Curitiba: PUCPRess, 2015.

SCHAUBER, S. K. et. al. The role of environmental and individual characteristics in the development of student achievement: a comparison between a traditional and a problem-based-learning curriculum. Advances in Health Sciences Education October 2015, Volume 20, Issue 4, pp 1033-1052. Disponível em: <http://link.springer.com/article/10.1007\%2Fs10459-015-9584-2> Acesso em: 8 jul. 2016.

SIEGEL, M. A. Filling in the Distance Between Us: Group Metacognition During Problem Solving in a Secondary Education Course. Journal of Science Education and Technology June 2012, Volume 21, Issue 3, pp 325-34. Disponível em: <http://link.springer.com/article/10.1007\%2Fs10956-011-9326-z> Acesso em: 5 jul. 2016.

STEFANOU, C. et. al. Self-regulation and autonomy in problem- and project-based learning environments. Active Learning in Higher Education July 2013 vol. 14 no. 2 109-122. Disponível em: <http://alh.sagepub.com/content/14/2/109.abstract> Acesso em: 8 jul. 2016.

TRINDADE, R.; COSME, A. Escola, Educação e Aprendizagem: desafios e respostas pedagógicas. - RJ: WAK Ed., 2010. 
VALLI, L. Listening to other voices: a description of teacher reflection in the United States. Peabody Journal of Education Volume 72, Issue 1, 1997. Disponível em: <http://www.tandfonline. com/doi/abs/10.1207/s15327930pje7201_4> Acesso em: 20 jul. 2016.

VIGOTSKI, L. S. A formação social da mente: o desenvolvimento dos processos psicológicos superiores. - 6a Edição - São Paulo: Martins Fontes, 1998.

WEBB, N. M.; MASTERGEORGE, A. M. The development of student's learning in peer-directed small groups. Cognition and Instruction, v21 n4 p361-428 2003. Disponível em: <http:// eric.ed.gov/?id=EJ761567> Acesso em: 20 jul. 2016.

\section{Notas}

${ }^{1} \mathrm{O}$ paradigma da instrução, segundo Trindade e Cosme (2010), privilegia a relação entre os professores e o saber, restando aos alunos uma posição nula ou receptiva do conteúdo.

${ }^{2}$ Segundo Claxton (2005) uma das principais estratégias ativas da compreensão é mobilizar ao máximo conhecimento e experiências prévias, como redes de significado preexistentes, para que o sujeito possa buscar ou criar a integração coerente dessas informações que serão base para a sua compreensão.

${ }^{3} \mathrm{O}$ ciclo da aprendizagem metacognitiva, proposta por Flavell (1979), inicia com uma tarefa e meta auto imposta ou externamente estabelecida; o conhecimento metacognitivo sobre essa classe de metas resgata a experiência metacognitiva de que a meta será difícil (ou não) de alcançar. Esses conhecimentos levam à seleção da estratégia cognitiva mais adequada, que desencadeia novas experiências metacognitivas sobre o desempenho na tarefa. Essas experiências, em combinação ao conhecimento metacognitivo, levam a estratégias de avaliação do empreendimento. Todo esse mapeamento levanta as dificuldades e retoma a ativação do conhecimento metacognitivo, e assim o ciclo recomeça até a conclusão da tarefa (FLAVELL, 1979).

* Mestranda em Educação pela Pontifícia Universidade Católica do Paraná, Curitiba, Paraná, Brasil.

* Professora doutora da Pontifícia Universidade Católica do Paraná, Curitiba, Paraná, Brasil.

\section{Correspondência}

Evelise Maria Labatut Portilho - Pontifícia Universidade Católica do Paraná, Departamento de Educação. Rua Imaculada Conceicao, 1155, Prado Velho. CEP: 80215901. Curitiba, Paraná, Brasil.

E-mail: adri.adri.v@gmail.com - eveliseportilho@gmail.com

Recebido em 21 de maio de 2016

Aprovado em 19 de julho de 2016 